\title{
Diffuse Large B-Cell Lymphoma Trans-differentiating into CD30 Positive Anaplastic Large Cell-like Lymphoma
}

\author{
Qiu JJ , Bhattacharyya S, Grant M and Bains A* \\ Department of Pathology, Temple University Hospital, \\ USA
}

*Correspondling author: Bains A, Department of Pathology, Temple University Hospital, Philadelphia, PA 19140, USA

Received: May 11, 2017; Accepted: J une 12, 2017; Published: J uly 06, 2017

\begin{abstract}
Post-Transplant Lymphoproliferative Disorders (PTLD) is mostly of B-cell origin. "Composite" PTLD with diffuse large B-cell lymphoma (DLBCL) and T/ anaplastic large cell lymphoma component are extremely rare. We report a case of PTLD after heart transplant with two distinct components. The patient was initially diagnosed with monomorphic PTLD/DLBCL, EBV negative involving the intestinal wall, occurring nine years after heart transplantation. Sixteen years post-transplant, the lymphoma recurred involving the intestine and showed two distinct components: DLBCL and anaplastic large cell lymphoma with strong homogeneous expression of CD45 and CD30 while lacking B- and T-cell lineage markers mimicking Anaplastic Large Cell Lymphoma (ALCL), ALK negative with a null-cell phenotype and TIA-1 expression. At this time, diffuse lymphadenopathy was also present and a jugular lymph node biopsy prior to the intestinal resection, purely demonstrated only the anaplastic large lymphoma cells with a null-cell phenotype leading to the diagnosis of ALCL, ALK negative. However, molecular studies performed on the anaplastic large cell component identified strongly positive clonal $\mathrm{lgH}$ gene rearrangement, identical to the prior lymphoma. Extensive sampling from the intestinal resection specimen identified a transitioning phase between two morphologically and immunohistochemically separate components. This is a unique case of PTLD which demonstrates an immunophenotypic lineage plasticity of the neoplastic cells over an extended post-transplant period.
\end{abstract}

Keywords: Post-transplant lymphoproliferative disorder; Diffuse large B-cell lymphoma; Anaplastic large cell lymphoma

\section{Case Presentation}

\section{Clinical features}

A 66-year-old male underwent heart transplant secondary to dilated cardiomyopathy and received tacrolimus for immunosuppressive therapy. Nine years post-transplant, he developed monomorphic PTLD/DLBCL (stage 1E with no evidence of bone marrow involvement) causing small intestinal obstruction which was treated with segmental resection and reduction of immunosuppressive medication. Subsequently the patient received complete remission with no evidence of lymphomatous involvement on PET/CT scan which lasted for two years until he presented with abdominal pain. The lymphoma recurred at the anastomotic site two years from the initial diagnosis, for which the patient underwent a hemicolectomy and four cycles of cyclophosphamide, doxorubicin, vincristine, and prednisone with rituximab (CHOP/R-CHOP) Sixteen years post-transplant, lymphoma recurred again, this time with extensive lymphadenopathy in addition to small intestinal involvement (Figure 1A and 1B). The complete blood count was unremarkable except for a low hemoglobin level $(5,700 \mathrm{~K} / \mu \mathrm{L}$ white blood count with normal differential, $-10.8 \mathrm{gm} / \mathrm{dL}$ hemoglobin, and $247 \mathrm{~K} / \mu \mathrm{L}$ platelets). Given the diffuse lymphadenopathy, initially a core biopsy of the most accessible jugular lymph node was performed which was diagnosed as ALCL, ALK negative with a null-cell phenotype. Subsequent to the diagnosis, the patient underwent partial small intestine resection to prevent potential perforation during treatment. A bone marrow biopsy was negative for lymphoma involvement. He then received salvage chemotherapy with six cycles of modified R-CHOP with addition of etoposide and achieved a complete response. It was followed by autologous stem cell transplant. At the time of this report, the patient has shown no signs of recurrent lymphoma.

\section{Pathologic features}

The initial lymphoma presented 9 years post-transplant as an exophytic lesion involving the ileum. The neoplastic infiltrate was composed of large cells with ovoid to irregular nuclei, vesicular chromatin, multiple central or membrane bound nucleoli and scant to moderate cytoplasm (Figure $2 \mathrm{~A}$ ). Immunohistochemical staining revealed diffuse positivity for LCA and CD20 (Figure 2B). The neoplastic cells were negative for CD10, BCL6, cyclin D1, and CD3. The proliferative index (Ki-67) was 60\%-70\%.

The first recurrence presented as a $6 \mathrm{~cm}$ polypoid mass in ileocolonic junction at the anastomotic site, with thickening of underlying submucosal tissue. H\&E sections showed monomorphic large neoplastic cells in sheets with occasional cells showing anaplastic morphology (Figure 2C). The tumor cells were positive for LCA, CD79a, CD22 and PAX5, while predominantly negative for CD20 (Figure 2D). A smaller subset (approximately 20-30\%) was positive for CD30, but negative for MUM1 and BCL2.

At second recurrence, sixteen years post-transplant, the
Citation: Qiu JJ, Bhattacharyya S, Grant M and Bains A. Diffuse Large B-Cell Lymphoma Trans-differentiating into CD30 Positive Anaplastic Large Cell-like Lymphoma. Ann Hematol Oncol. 2017; 4(7): 1162. 


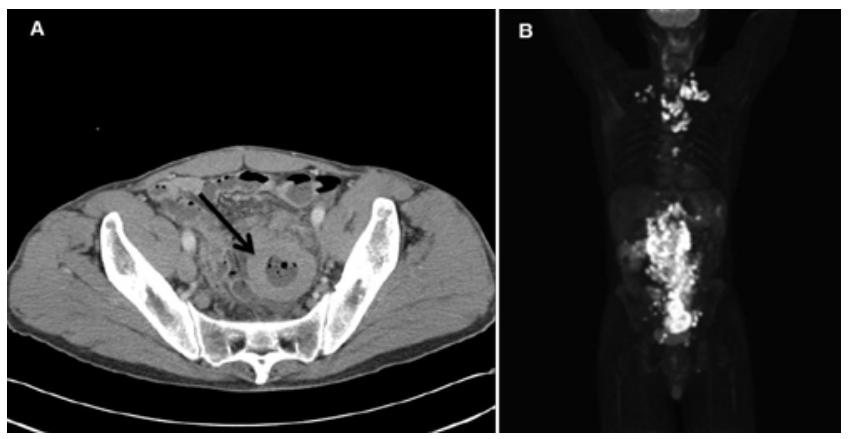

Figure 1: Radiologic features of the second recurrent intestinal DLBCL. (A) CT scan showing thickened intestinal wall. (B) PET/CT scan showing highly metabolically active small bowel wall and highly metabolically active adenopathy on both sides of the diaphragm.
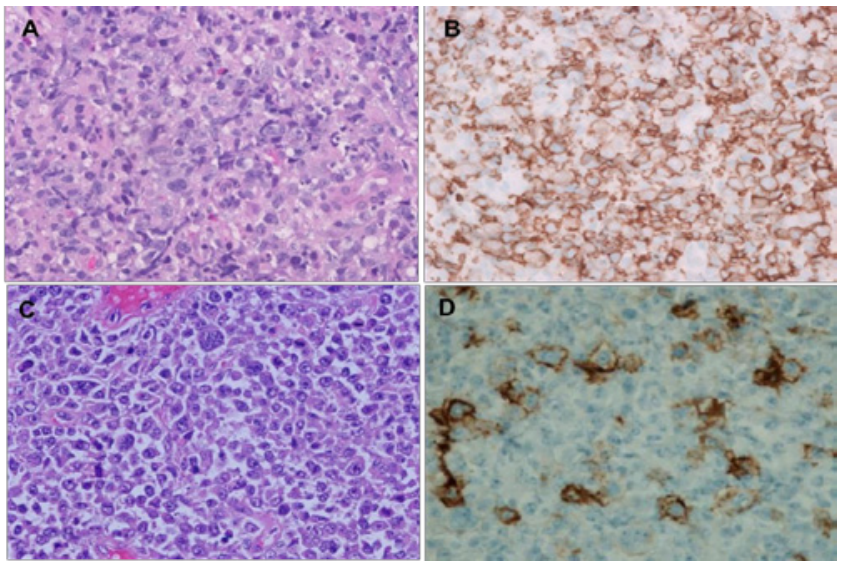

Figure 2: H\&E stains of initial DLBCL (A) and the first recurrent lymphoma (C). The initial neoplastic cells were positive for CD20 (B), while majority of neoplastic cells at the first recurrence were negative for CD20 (D) (magnification $x 400$ ).
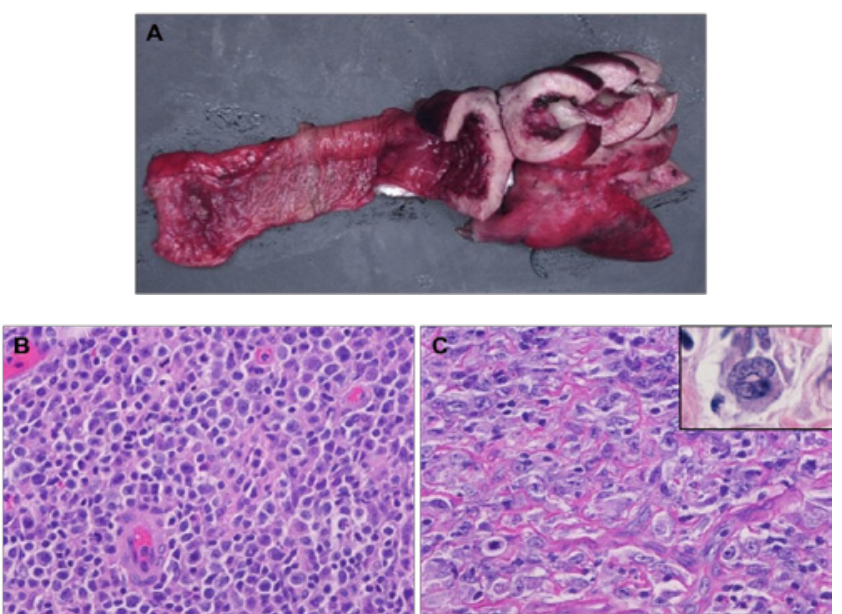

Figure 3: Gross and histopathologic features of the second recurrent lymphoma. (A) Small intestine showing circumferential thickened wall. Two components with distinct morphology, one similar to previous DLBCL $(B)$ and the second one with anaplastic morphology with scattered hallmark cells (C; inset) (magnification $\mathrm{x}$ 400).

disease was extensive with diffuse lymphadenopathy and intestinal involvement. A core biopsy from the left jugular lymph node was obtained first and demonstrated numerous large neoplastic cells with atypical mitotic figures, occasional cells with pleomorphic horseshoe shaped nuclei and moderately abundant cytoplasm. Hodgkin Reed Sternberg (HRS) cells were not seen. The neoplastic cells showed a null-cell immunophenotype similar to the bulky tumor in small intestine (resected subsequently). LCA and CD30 were strongly and homogeneously positive, while all remaining markers, including Band $\mathrm{T}$-cell lineage associated antigens were negative (CD20, PAX5, CD79a, CD22, CD10, BCL6, MUM1, CD138, CD2, CD3, CD4, CD5, CD7, CD8, CD15, EMA, and ALK1). In-situ hybridization studies for 


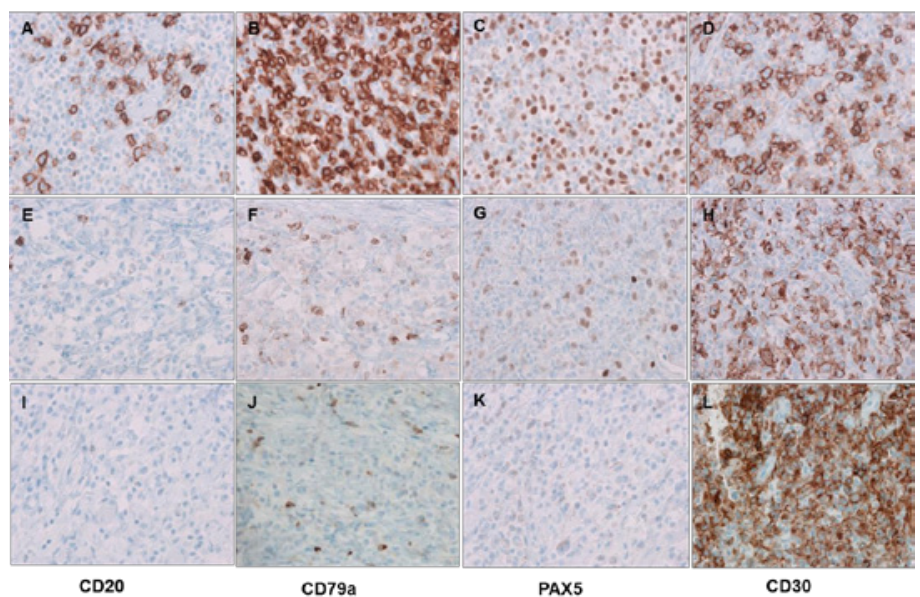

Figure 4: Immunophenotypic features of the second recurrent lymphoma. Two distinct components with distinct immunohistochemical patterns, one component similar to previous DLBCL (A-D), the second one with anaplastic morphology (I-L) and the transitioning component (E-H) (magnification $x 400)$

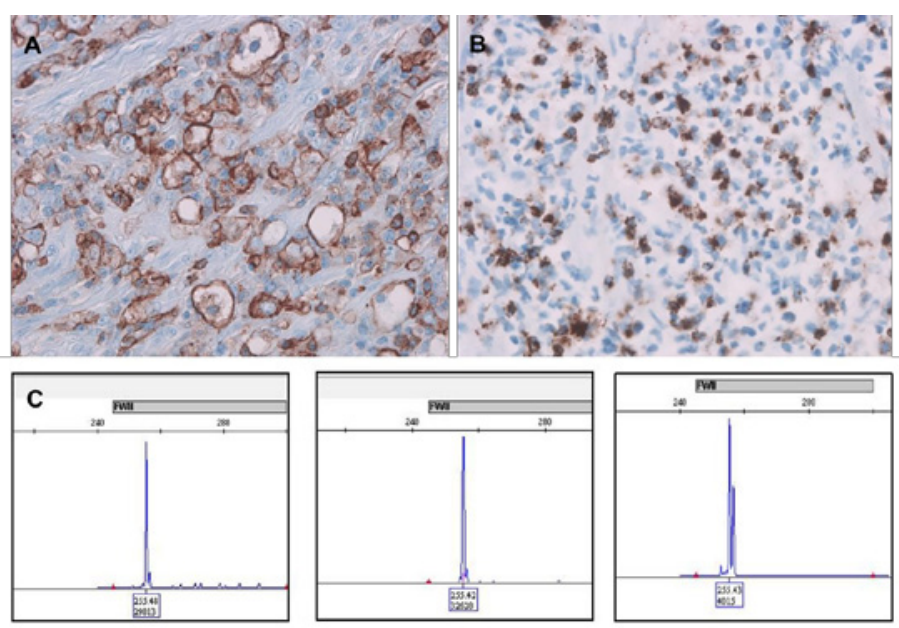

Figure 5: Homogeneous CD45 expression (A) and dot-like TIA-1 expression by anaplastic cells (B). IgH gene rearrangement studies on intestinal masses from both recurrences at 11 and 16 years post-transplant and jugular lymph node identifying a monoclonal peak at 255 bp at framework II in all three specimens (C).

EBV-encoded small RNA (EBER) were negative for all specimens. At this time, it was diagnosed as ALCL, ALK negative.

Small intestine showed circumferential thickening of the wall $(1.1 \mathrm{~cm}-6.7 \mathrm{~cm})$, with a homogeneously yellow-tan, fleshy cut surface (Figure 3A). Many subserosal/submucosal nodules $(0.3 \mathrm{~cm}-1.9 \mathrm{~cm})$ were also present. H\&E sections and immunohistochemical stains demonstrated two distinct components. The submucosal nodules (first component) demonstrated homogeneous sheets of intermediate to large sized lymphoid cells with centroblastic morphology and immunophenotype similar to patient's prior lymphoma recurrence in 2008 (Figure 3B and 3C). Here most neoplastic lymphocytes were positive for CD79a (Figure 4A and 4B) and PAX5 (Figure 4C), whereas only a minority subset (approximately $5 \%$ ) showed positivity for CD20. A subpopulation of these cells $(\sim 30 \%)$ was also strongly positive for CD30 (Figure 4D). A lambda light chain restriction was identified by in-situ hybridization. The serosal bulky mass (second component) displayed highly pleomorphic to anaplastic cells with many atypical mitotic figures and scattered horseshoe shaped hallmark cells; classic HRS cells were not seen. These neoplastic cells were negative for B-cell lineage associated antigens CD20 (Figure 4E and 4I), CD79a (Figure 4F and 4J), and CD22. Additional sampling identified occasional foci with transitioning expression of PAX5 from weak/heterogeneous staining to completely absent (Figure $4 \mathrm{G}$ and $4 \mathrm{~K})$. However, a strong and uniform expression of LCA and CD30 (Figure $4 \mathrm{H}$ and $4 \mathrm{~L}$ ) was present. There was no T-cell lineage defining antigen expression by the neoplastic cells. However, the anaplastic cells were positive for TIA1 with a dot-like granular expression pattern (Figure 5A and 5B), but negative for granzyme-B and ALK1.

Retrospective B-cell clonality study by IgH PCR on the intestinal masses from first and current recurrences and jugular lymph node demonstrated a clonal peak at 255 base pairin framework region II (Figure 5C). T-cell gene rearrangement studies showed no interpretable fragments for the mass of first recurrence, few oligoclonal peaks in TCR-gamma region and a polyclonal pattern for T-beta gene for the mass of the second recurrence, and a polyclonal TCR-beta and gamma gene rearrangement for the jugular lymph node. Final diagnosis was monomorphic PTLD: DLBCL with dedifferentiation 
to CD30 positive large cell non-Hodgkin lymphoma, EBV negative.

\section{Discussion}

This is an unusual case of PTLD with features not previously reported in the literature. There is a dedifferentiation of patient's previously diagnosed DLBCL toward a CD30 positive large cell lymphoma with loss of its lineage defining markers, mimicking an anaplastic large cell lymphoma, ALK negative (null-cell phenotype). However, a clear morphologic transition on sections from the resected small intestine with focally retained weak PAX5 expression in anaplastic large cells and a persistent B-cell clonality in the jugular lymph node identifies a link between the two separate neoplastic populations and supports a common B-cell origin. Similar to the of PTLD reported by Fortune et al., the DLBCL and T/Anaplastic large cell lymphoma components of a unique "composite" lymphoma, this case also suggests that there is a common B-cell precursor rather than a true composite lymphoma [1].

In the current case, the transition is gradual and initially observed at the first recurrence, with a smaller subset (approximately 20\%$30 \%$ ) showing positivity for CD30, CD79a, PAX5 and negativity for CD20. Interestingly, the loss of CD20 occurred before the patient received chemotherapy and rituximab. Further transition is evident in the second recurrence, with presence of two markedly distinct components. The bulk of lymphoma was diffusely positive for CD30 and CD45 while negative for CD15 and B- and T-cell markers. Known CD20-negative variants of DLBCL include primary effusion lymphoma, plasmablastic lymphoma, anaplastic lymphoma kinasepositive DLBCL and large B-cell lymphoma arising in human herpes virus 8 -associated multicentric Castleman disease [2,3]. Weak or negative CD20 levels have a significant negative impact on the survival of patients with DLBCL [4,5]. PEL, PBL and anaplastic lymphoma kinase-positive DLBCL are variably positive for CD30 [2]. However, it is not clear if there is a causal relationship between expression of CD30 and the loss of CD20.

CD30 was originally identified as a marker of Hodgkin and ReedSternberg cells in classical Hodgkin lymphoma [6]. CD30 was found to be variably positive in $81 \%$ of PTLD/DLBCL [7] and in about $14 \%$ of immunocompetent patients with DLBCL [8]. CD30 expression is reported to be associated with a favorable outcome in PTLD/DLBCL, regardless of EBV status [7]. In a large cohort of immunocompetent patients with DLBCL, CD30 expression was also demonstrated to be associated with favorable outcome. CD30 expression correlates with a unique gene expression signature suggesting a distinct molecular basis for its favorable outcome. However, the combination of CD30 and EBV expression in immunocompetent patients was correlated with an extremely aggressive clinical course [8]. The current case is a refractory PTLD, which usually has poor prognosis [9]. However, the patient has shown no signs of recurrent lymphoma for three years after last small bowel resection followed by salvage chemotherapy (modified R-CHOP with addition of etoposide) and autologous stem cell transplant. In an EBV negative PTLD, the transition to CD30 positive lymphoma may contribute to the favorable outcome. CD30 is used as a therapeutic target for brentuximab vedotin in ongoing successful clinical trials, which may further improve the outcome of CD30 positive lymphomas $[8,10]$.
Another interesting finding in this case is the aberrant expression of T-cell intracellular antigen-1 (TIA-1) in anaplastic cells with a granular expression pattern. TIA-1 is a cytotoxic granule predominantly expressed by cytotoxic CD8 positive T-cells and natural killer cells. The case reported by Fortune et al also showed aberrant expression of TIA-1 in anaplastic cells [1]. One study showed five of nine cases of hairy cell leukemia were positive for cytoplasmic TIA-1, with a small, dot-like, granular expression pattern, while 94 B-cell lymphomas of other types were negative for TIA-1. Based on these findings, TIA-1 reactivity in lymphomas does not necessarily indicate a T- or NK-cell derivation [11]. Neoplastic cells in $13.7 \%$ of stage-IIIB/IV Hodgkin's lymphoma also showed expression of TIA1 [12]. TIA-1 expression in DLBCL has previously not been reported.

Lastly, committed B-lymphoid cells are shown, particularly in mouse models, to be able to regain multi lineage potential and to transdifferentiate into non-B-lineage cells, challenging the rigid view of hematopoietic cellular differentiation [17]. It has been suggested that PAX5 expression determines B-cell commitment; however, even rearrangements at the IgH chain loci by itself are not signs of B-cell commitment [18]. PAX5 has been proposed to act downstream of E2A and EBF transcription factors and considered to be essential for commitment to and maintenance of the B-cell phenotype $[19,20]$. The current case is an in-vivo example demonstrating dedifferentiation and lineage plasticity of previously committed neoplastic hematopoietic B-cells toward a null-phenotype. It is unclear, although likely, that loss of PAX5 and potentially an imbalanced transcriptional factor activity in the presence of long standing and iatrogenically induced immune dysregulatory state and/or tumor microenvironment are a contributing factor for the observed transition in this case.

\section{Conclusion}

This is a unique case of EBV negative PTLD with a clear transition of DLBCL toward a CD30 positive large cell lymphoma mimicking anaplastic large cell lymphoma, ALK negative with null-cell phenotype. However, focally retained PAX5 expression in transitioning anaplastic cells and persistently same B-cell clonal peaks over an extended period of remissions and relapses, support a common B-cell origin.

\section{References}

1. Sabattini E, Bacci F, Sagramoso C, Pileri SA. WHO classification of tumoursof haematopoietic and lymphoid tissues in 2008: an overview. Pathologica. 2010; 102: 83-87.

2. Clarke CA, Morton LM, Lynch C, Pfeiffer RM, Hall EC, et al. Risk of lymphoma subtypes after solid organ transplantation in the United States. Br J Cancer. 2013; 109: 280-288.

3. Loren AW, Porter DL, Stadtmauer EA, Tsai DE. Post-transplant lymphoproliferative disorder: a review. Bone Marrow Transplant. 2003; 31: 145-155.

4. Al-Mansour Z, Nelson BP, Evens AM. Post-transplant lymphoproliferative disease (PTLD): risk factors, diagnosis, and current treatment strategies. Curr Hematol Malig Rep. 2013; 8: 173-183.

5. La Fortune K, Zhang D, Raca G, Ranheim EA. A Unique "Composite" PTLD with Diffuse Large B-Cell and T/Anaplastic Large Cell Lymphoma Components Occurring 17 Years after Transplant. Case Rep Hematol. 2013: 386147.

6. Castillo JJ, Chavez JC, Hernandez-Ilizaliturri FJ, Montes-Moreno S. CD20negative diffuse large B-cell lymphomas: biology and emerging therapeutic 
options. Expert Rev Hematol. 2015; 8: 343-354.

7. Li J, Zhao S, Wang J, Chen J, Wen W, et al. CD20-negative diffuse large B cell lymphoma: a comprehensive analysis of 695 cases. Tumour Biol. 2016 37: 3619-3637.

8. Suzuki Y, Yoshida T, Wang G, Togano T, Miyamoto S, et al. Association of CD20 levels with clinicopathological parameters and its prognostic significance for patients with DLBCL. Ann Hematol. 2012; 91: 997-1005.

9. Johnson NA, Boyle M, Bashashati A, Leach S, Brooks-Wilson A, et al. Diffuse large B-cell lymphoma: reduced CD20 expression is associated with an inferior survival. Blood. 2009; 113: 3773-3780.

10. Durkop H, Latza U, Hummel M, Eitelbach F, Seed B, et al. Molecular cloning and expression of a new member of the nerve growth factor receptor family that is characteristic for Hodgkin's disease. Cell. 1992; 68: 421-427.

11. Vase MO, Maksten EF, Bendix K, Hamilton-Dutoit S, Andersen C, et al Occurrence and prognostic relevance of CD30 expression in post-transplant lymphoproliferative disorders. Leuk Lymphoma. 2015; 56: 1677-1685.

12. Hu S, Xu-Monette ZY, Balasubramanyam A, Manyam GC, Visco C, et al CD30 expression defines a novel subgroup of diffuse large B-cell lymphoma with favorable prognosis and distinct gene expression signature: a report from the International DLBCL Rituximab-CHOP Consortium Program Study. Blood. 2013; 121: 2715-2724
13. Zimmermann $\mathrm{H}$, Trappe RU. Therapeutic options in post-transplant lymphoproliferative disorders. TherAdvHematol. 2011; 2: 393-407.

14. Kumar A, Younes A. Role of CD30 targeting in malignant lymphoma. Curr Treat Options Oncol. 2014; 15: 210-225.

15. Mori N, Murakami YI, Shimada S, Iwamizu-Watanabe S, Yamashita Y, et al. TIA-1 expression in hairy cell leukemia. Mod Pathol. 2004; 17: 840-846.

16. Camilleri-Broet S, Ferme C, Berger F, Lepage $E$, Bain S, et al. TiA1 in advanced-stage classical Hodgkin's lymphoma: no prognostic impact for positive tumour cells or number of cytotoxic cells. Virchows Arch. 2004; 445: 344-346.

17. Mathas S. The pathogenesis of classical Hodgkin's lymphoma: a model for B-cell plasticity. HematolOncolClin North Am. 2007; 21: 787-804.

18. Rolink AG, Schaniel C, Bruno L, Melchers F. In vitro and in vivo plasticity of Pax5-deficient pre-B I cells. ImmunolLett. 2002; 82: 35-40.

19. Busslinger M. Transcriptional control of early B cell development. Annu Rev Immunol. 2004; 22: 55-79.

20. Rolink AG, Schaniel C, Bruno L. In vitro and in vivo plasticity of Pax5-deficient pre-B I cells. Immunol Lett. 2002; 82: 35-40.
Ann Hematol Oncol - Volume 4 Issue 7 - 2017

ISSN : 2375-7965 | www.austinpublishing group.com

Bains et al. (C) All rights are reserved
Citation: Qiu JJ, Bhattacharyya S, Grant M and Bains A. Diffuse Large B-Cell Lymphoma Trans-differentiating into CD30 Positive Anaplastic Large Cell-like Lymphoma. Ann Hematol Oncol. 2017; 4(7): 1162. 